\title{
THE BLOCH SPACE AND BESOV SPACES OF ANALYTIC FUNCTIONS
}

\author{
KAREL STROETHOFF
}

We shall give an elementary proof of a characterisation for the Bloch space due to Holland and Walsh, and obtain analogous characterisations for the little Bloch space and Besov spaces of analytic functions on the unit disk in the complex plane.

\section{INTRODUCTION}

We let $\mathbb{D}=\{z \in \mathbb{C}:|z|<1\}$, and use $d A$ for the normalised Lebesgue area measure on $\mathbb{D}$. For $1<p<\infty$, an analytic function $f$ is in the Besov space $\mathcal{B}_{p}$ if

$$
\|f\|_{\mathcal{B}_{p}}:=\left(\int_{\mathbb{D}}\left|f^{\prime}(z)\right|^{p}\left(1-|z|^{2}\right)^{p-2} d A(z)\right)^{1 / p}<\infty
$$

that is, the function $\left(1-|z|^{2}\right) f^{\prime} \in L^{p}(\mathbb{D}, d \lambda)$, where $d \lambda(z)=\left(1-|z|^{2}\right)^{-2} d A(z)$. We note that the measure $\lambda$ is not a finite measure on $\mathbb{D} ;$ its importance stems from the fact that it is Möbius-invariant. To make this precise we need more notation. For $w \in \mathbb{D}$ the Möbius transformation $\varphi_{w}$ is defined by

$$
\varphi_{w}(z)=\frac{w-z}{1-\bar{w} z}, \text { for } z \in \mathbb{D} .
$$

The following identity is easily verified:

$$
1-\left|\varphi_{w}(z)\right|^{2}=\frac{\left(1-|w|^{2}\right)\left(1-|z|^{2}\right)}{|1-\bar{w} z|^{2}} .
$$

So, the function $\varphi_{w}$ maps $\mathbb{D}$ into itself. It is furthermore easy to verify that $\varphi_{w}$ is its own inverse. Noting that $\varphi_{w}^{\prime}(z)=\left(|w|^{2}-1\right) /(1-\bar{w} z)^{2}$, the above identity states:

$$
\left(1-|z|^{2}\right)\left|\varphi_{w}^{\prime}(z)\right|=1-\left|\varphi_{w}(z)\right|^{2},
$$

Received 25th October, 1995

The author ackowledges partial support through summer grants from the University of Montana and the Montana University System.

Copyright Clearance Centre, Inc. Serial-fee code: 0004-9729/96 \$A2.00+0.00. 
and thus $d \lambda\left(\varphi_{w}(z)\right)=\left(1-\left|\varphi_{w}(z)\right|^{2}\right)^{-2}\left|\varphi_{w}^{\prime}(z)\right|^{2} d A(z)=\left(1-|z|^{2}\right)^{-2} d A(z)=d \lambda(z)$. Hence we have the following change-of-variable formula:

$$
\int_{\mathbb{D}} h\left(\varphi_{w}(z)\right) d \lambda(z)=\int_{\mathbb{D}} h(u) d \lambda(u),
$$

where $h$ is a positive measurable function on $\mathbb{D}$. Using (3) it is easily seen that $\left\|f \circ \varphi_{w}\right\|_{\mathcal{B}_{p}}=\|f\|_{B_{p}}$, and consequently, if $f \in \mathcal{B}_{p}$, then $f \circ \varphi_{w} \in \mathcal{B}_{p}$, for all $w \in \mathbb{D}$. The Besov space $\mathcal{B}_{1}$ is defined differently: it is the set of all analytic functions $f$ on $\mathbb{D}$ such that

$$
\|f\|_{B_{1}}:=\int_{\mathbb{D}}\left|f^{\prime \prime}(z)\right| d A(z)<\infty .
$$

Even though the above semi-norm is not Möbius-invariant, the Besov space $\mathcal{B}_{1}$ is; that is, if $f \in \mathcal{B}_{1}$, then $f \circ \varphi_{w} \in \mathcal{B}_{1}$, for all $w \in \mathbb{D}$. Another Möbius-invariant space of analytic functions on $\mathbb{D}$ is the Bloch space $\mathcal{B}$; it is the set of all analytic functions $f$ on D for which

$$
\|f\|_{B}:=\sup _{z \in \mathbb{D}}\left(1-|z|^{2}\right)\left|f^{\prime}(z)\right|<\infty .
$$

That $\mathcal{B}$ is Möbius-invariant is most easily seen from the observation that $\left(1-|z|^{2}\right)\left|f^{\prime}(z)\right|=\left|\left(f \circ \varphi_{z}\right)^{\prime}(0)\right|$, so that $\|f\|_{\mathcal{B}}=\sup \left\{\left|\left(f \circ \varphi_{z}\right)^{\prime}(0)\right|: z \in \mathbb{D}\right\}$, and thus $\left\|f \circ \varphi_{w}\right\|_{\mathcal{B}}=\|f\|_{\mathcal{B}}$, for all $w \in \mathbb{D}$. Note that $\mathcal{B}_{1} \subset \mathcal{B}_{p} \subset \mathcal{B}$ for each $1<p<\infty$. It is easy to prove that all these spaces are Banach spaces. Rubel and Timoney [9] have actually shown that the Bloch space $\mathcal{B}$ is maximal among all Möbius-invariant Banach spaces of analytic functions on $\mathbb{D}$ (provided there are so-called "decent" linear functionals). In [2] Arazy and Fisher have shown that the Besov space $\mathcal{B}_{1}$ is minimal among all Möbius-invariant Banach spaces of analytic functions on $\mathbb{D}$ (see also [3]). The Besov space $\mathcal{B}_{2}$, more often referred to as the Dirichlet space, is easily seen to be a Hilbert space. In [4] Arazy and Fisher proved that $\mathcal{B}_{2}$ is the only Möbius-invariant Hilbert space of analytic functions on $\mathbb{D}$.

In the next section we shall state and prove a criterion for containment in the Bloch space obtained by Holland and Walsh [8]. In section 3 we shall prove a characterisation for the analytic Besov spaces analogous to the criterion of Holland and Walsh for containment in the Bloch space.

\section{The Bloch Space}

In [8] Holland and Walsh obtained the following characterisation for the Bloch space.

THEOREM 1. For an analytic function $f$ on $\mathbb{D}$ :

$$
f \in \mathcal{B} \Longleftrightarrow \sup \left\{\left(1-|z|^{2}\right)^{1 / 2}\left(1-|w|^{2}\right)^{1 / 2}\left|\frac{f(z)-f(w)}{z-w}\right|: z, w \in \mathbb{D}, z \neq w\right\}<\infty
$$


Holland and Walsh's proof of the above result is quite complicated. We shall show how the Möbius-invariance can be exploited to obtain a very easy proof of the above theorem.

Proof of Theorem 1: The implication " $\Leftarrow$ " is trivial. To prove the other implication, suppose that $f \in \mathcal{B}$. Then

$$
|f(u)-f(0)| \leqslant \int_{0}^{1}|u|\left|f^{\prime}(t u)\right| d t \leqslant\|f\|_{B} \int_{0}^{1} \frac{|u|}{1-t^{2}|u|^{2}} d t=\|f\|_{B} \frac{1}{2} \log \frac{1+|u|}{1-|u|},
$$

for each $u \in \mathbb{D}$. Now

$$
\begin{aligned}
\frac{1}{2} \log \frac{1+|u|}{1-|u|} & =\frac{1}{2} \log \frac{(1+|u|)^{2}}{1-|u|^{2}}=\log \frac{1+|u|}{\left(1-|u|^{2}\right)^{1 / 2}} \\
& \leqslant \frac{1+|u|}{\left(1-|u|^{2}\right)^{1 / 2}}-1=\frac{1+|u|-\left(1-|u|^{2}\right)^{1 / 2}}{\left(1-|u|^{2}\right)^{1 / 2}} \\
& \leqslant \frac{1+|u|-(1-|u|)}{\left(1-|u|^{2}\right)^{1 / 2}}=\frac{2|u|}{\left(1-|u|^{2}\right)^{1 / 2}}
\end{aligned}
$$

where we used the inequality $\left(1-|u|^{2}\right)^{1 / 2} \geqslant 1-|u|$. It follows that

$$
|f(u)-f(0)| \leqslant\|f\|_{B} \frac{2|u|}{\left(1-|u|^{2}\right)^{1 / 2}},
$$

for all $u \in \mathbb{D}$. For $z, w \in \mathbb{D}$, replace $f$ in the above inequality by $f \circ \varphi_{w}$ and let $u=\varphi_{w}(z)$. Using $\varphi_{w}\left(\varphi_{w}(z)\right)=z,\left\|f \circ \varphi_{w}\right\|_{B}=\|f\|_{\mathcal{B}}$ and identity (1), we get

$$
|f(z)-f(w)| \leqslant\|f\|_{\mathcal{B}} \frac{2\left|\varphi_{w}(z)\right|}{\left(1-\left|\varphi_{w}(z)\right|^{2}\right)^{1 / 2}}=2\|f\|_{B} \frac{|z-w|}{\left(1-|z|^{2}\right)^{1 / 2}\left(1-|w|^{2}\right)^{1 / 2}},
$$

and thus

$$
\left(1-|z|^{2}\right)^{1 / 2}\left(1-|w|^{2}\right)^{1 / 2}\left|\frac{f(z)-f(w)}{z-w}\right| \leqslant 2\|f\|_{B},
$$

for distinct $z, w \in \mathbb{D}$, completing the proof of Theorem 1 .

Before we consider the analytic Besov spaces in the next section, we briefly discuss the little Bloch space $\mathcal{B}_{0}$, the set of all analytic functions $f$ on $\mathbb{D}$ for which

$$
\lim _{|z| \rightarrow 1^{-}}\left(1-|z|^{2}\right) f^{\prime}(z)=0
$$


For an analytic function $f$ on $\mathbb{D}$ and $0<t<1$ the dilate $f_{t}$ is the function defined by $f_{t}(z)=f(t z)$. In [1] it is shown that for an analytic function $f$ on $\mathbb{D}: f \in \mathcal{B}_{0}$ if and only if $\left\|f-f_{t}\right\|_{\mathcal{B}} \rightarrow 0$ as $t \rightarrow 1^{-}$.

In analogy to Theorem 1 we have the following result.

ThEOREM 2. For an analytic function $f$ on $\mathbb{D}$ :

$f \in \mathcal{B}_{0} \Longleftrightarrow \lim _{|z| \rightarrow 1^{-}} \sup \left\{\left(1-|z|^{2}\right)^{1 / 2}\left(1-|w|^{2}\right)^{1 / 2}\left|\frac{f(z)-f(w)}{z-w}\right|: w \in \mathbb{D}, w \neq z\right\}=0$

Proof: The implication " $\Leftarrow$ " is again trivial. To prove the other implication, suppose that $f \in \mathcal{B}_{0}$ and let $0<t<1$. Using (4) we see that the dilate $f_{t}$ satisfies:

$$
\begin{aligned}
\left(1-|z|^{2}\right)^{1 / 2}\left(1-|w|^{2}\right)^{1 / 2}\left|\frac{f_{t}(z)-f_{t}(w)}{z-w}\right| & \leqslant 2 t \frac{\left(1-|z|^{2}\right)^{1 / 2}\left(1-|w|^{2}\right)^{1 / 2}}{\left(1-|t z|^{2}\right)^{1 / 2}\left(1-|t w|^{2}\right)^{1 / 2}}\|f\|_{B} \\
& \leqslant \frac{2 t}{1-t^{2}}\left(1-|z|^{2}\right)^{1 / 2}\|f\|_{B} .
\end{aligned}
$$

Applying inequality (4) to the function $f-f_{t}$, it follows with the help of the triangle inequality that

$$
\begin{aligned}
\sup \left\{\left(1-|z|^{2}\right)^{1 / 2}\left(1-|w|^{2}\right)^{1 / 2}\left|\frac{f(z)-f(w)}{z-w}\right|: w \in \mathbb{D}, w \neq z\right\} \\
\leqslant \frac{2 t}{1-t^{2}}\left(1-|z|^{2}\right)^{1 / 2}\|f\|_{\mathcal{B}}+2\left\|f-f_{t}\right\|_{\mathcal{B}} .
\end{aligned}
$$

First letting $|z| \rightarrow 1^{-}$and then $t \rightarrow 1^{-}$, the implication " $\Rightarrow$ " follows.

For various other characterisations of the Bloch and little Bloch space we refer the reader to $[1,5,10,11,12]$.

\section{Besov Spaces of Analytic Functions}

Another way of interpreting Theorem 1 is that for an analytic function $f$ on $\mathbb{D}$ we have $f \in \mathcal{B}$ if and only if the function $\left(1-|z|^{2}\right)^{1 / 2}\left(1-|w|^{2}\right)^{1 / 2}(f(z)-f(w)) /(z-w)$ is in $L^{\infty}(\mathbb{D} \times \mathbb{D})$. We shall prove the corresponding result for the Besov spaces:

THEOREM 3. If $2<p<\infty$, then for an analytic function $f$ on $\mathbb{D}$ we have:

$$
f \in \mathcal{B}_{p} \Longleftrightarrow \int_{\mathbb{D}} \int_{\mathbb{D}}\left|\frac{f(z)-f(w)}{z-w}\right|^{p}\left(1-|z|^{2}\right)^{p / 2}\left(1-|w|^{2}\right)^{p / 2} d \lambda(w) d \lambda(z)<\infty .
$$

Of special interest is the case in which $\left(1-|z|^{2}\right)^{p / 2} d \lambda(z)=d A(z)$. This is so when $p=4$, and we have the following special case of Theorem 3 : 
COROLlary 4. For an analytic function $f$ on $\mathbb{D}$ we have:

$$
f \in \mathcal{B}_{4} \Longleftrightarrow \int_{\mathbb{D}} \int_{\mathbb{D}}\left|\frac{f(z)-f(w)}{z-w}\right|^{4} d A(z) d A(w)<\infty
$$

In the proof of Theorem 3 we shall need a couple of lemmas. In order to state the first of these lemmas we need to introduce more notation.

For $w \in \mathbb{D}$ and $0<r<1$ we write $D(w, r)$ for the set $\left\{\varphi_{w}(z):|z|<r\right\}=\varphi_{w}(r \mathbb{D})$. Because $\varphi_{w}$ is a Möbius transformation, the set $D(w, r)$ is a Euclidean disk contained in $\mathbb{D}$. Its Euclidean centre and radius are easily determined to be $\left(1-r^{2}\right) w /\left(1-r^{2}|w|^{2}\right)$ and $\left(1-|w|^{2}\right) r /\left(1-r^{2}|w|^{2}\right)$ respectively (see, for example [7, Section 1.1]). We refer to the set $D(w, r)$ as the pseudohyperbolic disk centred at $w$ with (pseudohyperbolic) radius $\boldsymbol{r}$.

Lemma 5. Let $1 \leqslant p<\infty, \alpha>0$, and $0<r<1$. There is a constant $C$, only depending upon $p, \alpha$, and $r$, such that

$$
\int_{D(w, r)}|g(z)|^{p}\left(1-|z|^{2}\right)^{\alpha} d \lambda(z) \geqslant C|g(w)|^{p}\left(1-|w|^{2}\right)^{\alpha}
$$

for every analytic function $g$ on $\mathbb{D}$ and $w \in \mathbb{D}$.

Proof: Let $g$ be an analytic function on $\mathbb{D}$. It is easy to verify that

$$
\int_{r \mathbb{D}} g(z)\left(1-|z|^{2}\right)^{\alpha} d \lambda(z)=g(0) \int_{r \mathbb{D}}\left(1-|z|^{2}\right)^{\alpha} d \lambda(z)
$$

and by Jensen's inequality we have:

$$
\int_{D(0, r)}|g(z)|^{p}\left(1-|z|^{2}\right)^{\alpha} d \lambda(z) \geqslant|g(0)|^{p} \int_{D(0, r)}\left(1-|z|^{2}\right)^{\alpha} d \lambda(z)=C_{0}|g(0)|^{p}
$$

so the inequality holds for $w=0$ with $C_{0}=\int_{0}^{r^{2}}(1-x)^{\alpha-2} d x$. Now if $w \in \mathbb{D}$, apply the above inequality to $g \circ \varphi_{w}$. Using change-of-variable formula (3) and identity (1) we have:

$$
\begin{aligned}
C_{0}|g(w)|^{p} & \leqslant \int_{D(0, r)}\left|g\left(\varphi_{w}(z)\right)\right|^{p}\left(1-|z|^{2}\right)^{\alpha} d \lambda(z) \\
& =\int_{D(w, r)}|g(u)|^{p}\left(1-\left|\varphi_{w}(u)\right|^{2}\right)^{\alpha} d \lambda(u) \\
& \leqslant \int_{D(w, r)}|g(u)|^{p} \frac{\left(1-|u|^{2}\right)^{\alpha}\left(1-|w|^{2}\right)^{\alpha}}{|1-\bar{w} u|^{2 \alpha}} d \lambda(u) .
\end{aligned}
$$


If $u \in D(w, r)$, then $u=\varphi_{w}(z)$, for some $z \in r \mathbb{D}$, and thus $|1-\bar{w} u|$ $=\left(1-|w|^{2}\right) /|1-\bar{w} z| \geqslant\left(1-|w|^{2}\right) /(1+r)$. It follows that

$$
\begin{aligned}
\int_{D(w, r)}|g(u)|^{p} \frac{\left(1-|u|^{2}\right)^{\alpha}\left(1-|w|^{2}\right)^{\alpha}}{|1-\bar{w} u|^{2 \alpha}} d \lambda(u) & \\
& \leqslant \frac{(1+r)^{2 \alpha}}{\left(1-|w|^{2}\right)^{\alpha}} \int_{D(w, r)}|g(u)|^{p}\left(1-|u|^{2}\right)^{\alpha} d \lambda(u),
\end{aligned}
$$

proving the lemma (with $C=C_{0} /(1+r)^{2 \alpha}$ ).

Towards the Proof of Theorem 3. Let $f$ be analytic on $\mathbb{D}$. For $w \in \mathbb{D}$ write $D(w)$ for $D(w, 1 / 2)$, and apply Lemma 5 to the function $g(z)=(f(z)-f(w)) /(z-w)$ to get:

$$
\int_{D(w)}\left|\frac{f(z)-f(w)}{z-w}\right|^{p}\left(1-|z|^{2}\right)^{p / 2} d \lambda(z) \geqslant C\left(1-|w|^{2}\right)^{p / 2}\left|f^{\prime}(w)\right|^{p}
$$

It follows that

$$
\begin{aligned}
\int_{\mathbb{D}} \int_{\mathbb{D}}\left|\frac{f(z)-f(w)}{z-w}\right|^{p} & \left(1-|z|^{2}\right)^{p / 2}\left(1-|w|^{2}\right)^{p / 2} d \lambda(z) d \lambda(w) \\
& \geqslant \int_{\mathbb{D}} \int_{D(w)}\left|\frac{f(z)-f(w)}{z-w}\right|^{p}\left(1-|z|^{2}\right)^{p / 2}\left(1-|w|^{2}\right)^{p / 2} d \lambda(z) d \lambda(w) \\
& \geqslant C \int_{\mathbb{D}}\left|f^{\prime}(w)\right|^{p}\left(1-|w|^{2}\right)^{p} d \lambda(w) .
\end{aligned}
$$

This proves the implication " $\Leftarrow$ " in Theorem 3 . To prove the converse we shall use the following lemma.

Lemma 6. Let $1 \leqslant p<\infty$, and $\alpha>1$. There is a finite constant $C$, only depending on $p$ and $\alpha$ such that

$$
\int_{\mathbb{D}}\left|\frac{g(u)-g(0)}{u}\right|^{p}\left(1-|u|^{2}\right)^{\alpha} d \lambda(u) \leqslant C \int_{\mathbb{D}}\left|g^{\prime}(u)\right|^{p}\left(1-|u|^{2}\right)^{p+\alpha} d \lambda(u)
$$

for every analytic function $g$ on $\mathbb{D}$. 
ProOF: This follows easily from Theorem 5.6 of [6] and the fact that for the measure $d \mu(u)=\left(1-|u|^{2}\right)^{\alpha} d \lambda(u)$ the linear operator $V$, defined on $L^{p}(\mathbb{D}, d \mu)$ by $(V g)(u)=(g(u)-g(0)) / u$, is bounded (as can be shown using the closed graph theorem).

Completion of the Proof Theorem 3: Fix $w \in \mathbb{D}$. Using identity (1) and making the change-of-variables $z=\varphi_{w}(u)$ we have:

$$
\begin{aligned}
\int_{\mathbb{D}}\left|\frac{f(z)-f(w)}{z-w}\right|^{p} & \left(1-|z|^{2}\right)^{p / 2}\left(1-|w|^{2}\right)^{p / 2} d \lambda(z) \\
& =\int_{\mathbb{D}}\left|\frac{f(z)-f(w)}{\varphi_{w}(z)}\right|^{p}\left(1-\left|\varphi_{w}(z)\right|^{2}\right)^{p / 2} d \lambda(z) \\
& =\int_{\mathbb{D}}\left|\frac{\left(f \circ \varphi_{w}\right)(u)-\left(f \circ \varphi_{w}\right)(0)}{u}\right|^{p}\left(1-|u|^{2}\right)^{p / 2} d \lambda(u) .
\end{aligned}
$$

Applying Lemma 6 to the function $f \circ \varphi_{w}$ we see that

$$
\begin{array}{rlr}
\int_{\mathbb{D}}\left|\frac{\left(f \circ \varphi_{w}\right)(u)-\left(f \circ \varphi_{w}\right)(0)}{u}\right|^{p}\left(1-|u|^{2}\right)^{p / 2} d \lambda(u) & \\
& \leqslant C \int_{\mathbb{D}}\left|f^{\prime}\left(\varphi_{w}(u)\right)\right|^{p}\left|\varphi_{w}^{\prime}(u)\right|^{p}\left(1-|u|^{2}\right)^{p}\left(1-|u|^{2}\right)^{p / 2} d \lambda(u) & \\
& =C \int_{\mathbb{D}}\left|f^{\prime}\left(\varphi_{w}(u)\right)\right|^{p}\left(1-\left|\varphi_{w}(u)\right|^{2}\right)^{p}\left(1-|u|^{2}\right)^{p / 2} d \lambda(u) & \text { [using (2)] } \\
& =C \int_{\mathbb{D}}\left|f^{\prime}(z)\right|^{p}\left(1-|z|^{2}\right)^{p}\left(1-\left|\varphi_{w}(z)\right|^{2}\right)^{p / 2} d \lambda(z) & \text { [using (3)] } \\
& =C \int_{\mathbb{D}}\left|f^{\prime}(z)\right|^{p}\left(1-|z|^{2}\right)^{(3 / 2) p-2} \frac{\left(1-|w|^{2}\right)^{p / 2}}{|1-\bar{w} z|^{p}} d A(z) & \text { [using (1)]. }
\end{array}
$$

Integrating with respect to $w$ now yields:

$$
\begin{aligned}
\int_{\mathbb{D}} \int_{\mathbb{D}}\left|\frac{f(z)-f(w)}{z-w}\right|^{p}\left(1-|z|^{2}\right)^{p / 2}\left(1-|w|^{2}\right)^{p / 2} d \lambda(z) d \lambda(w) \\
\leqslant C \int_{\mathbb{D}} \int_{\mathbb{D}}\left|f^{\prime}(z)\right|^{p}\left(1-|z|^{2}\right)^{(3 / 2) p-2} \frac{\left(1-|w|^{2}\right)^{(p / 2)-2}}{|1-\bar{w} z|^{p}} d A(z) d A(w) \\
\quad=C \int_{\mathbb{D}}\left|f^{\prime}(z)\right|^{p}\left(1-|z|^{2}\right)^{(3 / 2) p-2} \int_{\mathbb{D}} \frac{\left(1-|w|^{2}\right)^{(p / 2)-2}}{|1-\bar{w} z|^{p}} d A(w) d A(z) .
\end{aligned}
$$


As a special case of one of the Forelli-Rudin estimates (see, for example, [12, Lemma 4.2.2]), the inner integral is bounded by a constant times $\left(1-|z|^{2}\right)^{-p / 2}$. (Note that this estimate requires $(p / 2)-2>-1$, that is, $p>2$.) Thus, there is a constant $C^{\prime}$ such that

$$
\begin{gathered}
\left.\int_{\mathbb{D}} \int_{\mathbb{D}} \frac{f(z)-f(w)}{z-w}\right|^{p}\left(1-|z|^{2}\right)^{p / 2}\left(1-|w|^{2}\right)^{p / 2} d \lambda(z) d \lambda(w) \\
\leqslant C^{\prime} \int_{\mathbb{D}}\left|f^{\prime}(z)\right|^{p}\left(1-|z|^{2}\right)^{(3 / 2) p-2}\left(1-|z|^{2}\right)^{-p / 2} d A(z) \\
=C^{\prime} \int_{\mathbb{D}}\left|f^{\prime}(z)\right|^{p}\left(1-|z|^{2}\right)^{p} d \lambda(z)
\end{gathered}
$$

and the proof of Theorem 3 is completed.

Remark. For $1 \leqslant p<\infty$ and $\alpha \leqslant-1$, an analytic function $h$ on $\mathbb{D}$ satisfies $\int_{\mathbb{D}}|h(z)|^{p}$ $\left(1-|z|^{2}\right)^{\alpha} d A(z)<\infty$ only when $h \equiv 0$. It is easily seen that for $1 \leqslant p \leqslant 2$ the condition of Theorem 3 involving the iterated integral implies that the function $f$ must be constant. Thus, the conclusion of Theorem 3 does not hold for $1 \leqslant p \leqslant 2$.

\section{REFERENCES}

[1] J.M. Anderson, J. Clunie and Ch. Pommerenke, 'On Bloch functions and normal functions', J. Reine Angew. Math. 270 (1974), 12-37.

[2] J. Arazy and S.D. Fisher, 'Some aspects of the minimal Möbius-invariant spaces of analytic functions on the unit disc', in Interpolation spaces and allied topics in analysis, (M. Cwikel and J. Peetre, Editors), Lecture Notes in Math, 1070 (Springer-Verlag, Berlin, Heidelberg, New York, 1984), pp. 24-44.

[3] J. Arazy and S.D. Fisher, 'The uniqueness of the Dirichlet space among Möbius-invariant Hilbert spaces', Illinois J. Math. 29 (1985), 449-462.

[4] J. Arazy, S.D. Fisher and J. Peetre, 'Möbius-invariant function spaces', J. Reine Angew. Math. 363 (1985), 110-145.

[5] S. Axler, 'The Bergman space, the Bloch space, and commutators of multiplication operators', Duke Math. J. 53 (1986), 315-332.

[6] P.L. Duren, Theory of $H^{p}$ spaces (Academic Press, 1970).

[7] J.B. Garnett, Bounded analytic functions (Academic Press, 1981).

[8] F. Holland and D. Walsh, 'Criteria for membership of Bloch space and its subspace BMOA', Math. Ann. 273 (1986), 317-335.

[9] L.A. Rubel and R. Timoney, 'An extremal property of the Bloch space', Proc. Amer. Math. Soc. 75 (1979), 45-49.

[10] K. Stroethoff, 'Besov-type characterisations for the Bloch space', Bull. Austral. Math. Soc. 39 (1989), 405-420. 
[11] K. Stroethoff, 'Nevanlinna-type characterizations for the Bloch space and related spaces', Proc. Edinburgh Math. Soc. 33 (1990), 123-141.

[12] K. Zhu, Operator theory in function spaces (Marcel Dekker, 1990).

Department of Mathematical Sciences

The University of Montana

Missoula MT 59812-1032

United States of America 